\title{
Eye-Tracking Research Special Session (Part 2): How to Design Attention- Grabbing Communications? An Abstract
}

\author{
Sophie Lacoste-Badie
}

\begin{abstract}
The goal of this session is to emphasize the role of attention grabbing during advertising exposure using eye-tracking devices in labs.

Hübner Barcelos, Correa Dantas, and Senecal investigate how the use of a human or a corporate tone of voice on hotels' brand pages influences information search and customer attitudes on social media. They performed an online pilot study and an eye-tracking experiment in a lab setting to test the effects of a human or corporate tone of voice on social media. This work contributes to the understanding of the branding of hospitality services on social media.

Baccino and Cherif conducted an eye-tracking study to investigate the effects of ads displayed in unpredictable locations on websites. Their experiment was design to verify when ads grab visual attention and when "banner blindness" occurs. Specifically, this study explores how attention varies according to the different levels of ads location unpredictability (low_skyscraper vs moderate_pop-up vs high_ slide-in) and ads animation (static vs animated).

Lacoste-Badie and Kacha examine the effect of color on attention devoted to advertisements displayed on print magazine. The study focuses on the impact of using "colors" and/or "black and white" in print advertisements on attention processes using a remote eye-tracking device, attitude toward the advertisement (Aad), and attitude toward the brand (Ab). Specifically, this study explores the contrast effect between four conditions: (1) color ad, (2) black and white ad, (3) product/ packshot in color with a black and white background ad, and (4) product/packshot in black and white with a background color ad.

Together, the three papers offer new light on the interest of eye-tracking technology to understand multiple aspects of consumer behavior, especially attentional processes during exposure to marketing stimuli in lab settings.
\end{abstract}

References Available Upon Request

\footnotetext{
S. Lacoste-Badie $(\triangle)$

Rennes 1 University (IUT GEA), Rennes, France

e-mail: sophie.lacoste-badie@univ-rennes1.fr
} 